\title{
De Novo Design of Selective Quadruplex-Duplex Junction Ligands and Structural Characterisation of Their Binding Mode: Targeting the G4 Hot-Spot
}

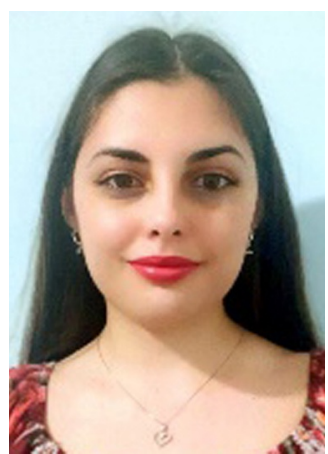

Laura Díaz-Casado

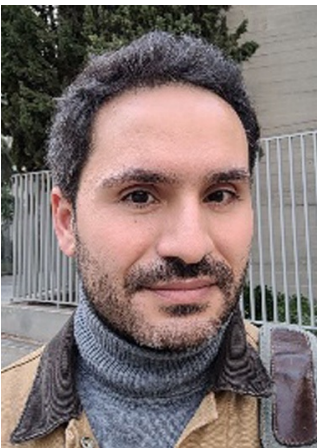

Andrés G. Santana

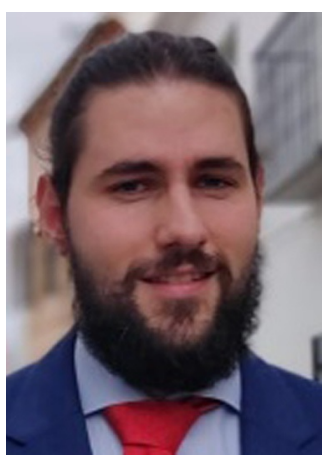

Israel Serrano-Chacón

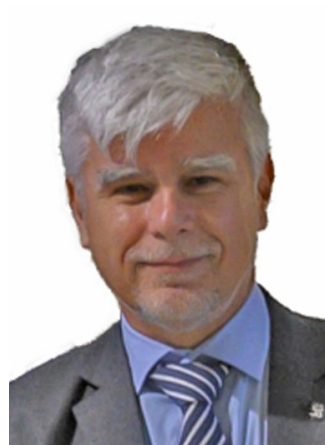

Carlos González

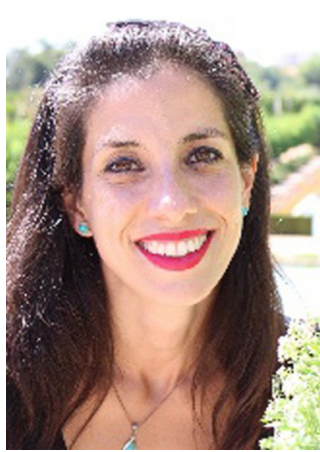

Laura Montalvillo-Jiménez

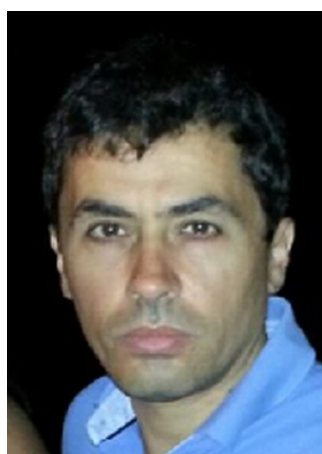

Juan Luis Asensio

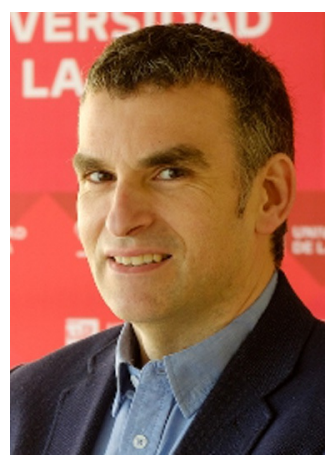

Francisco Corzana

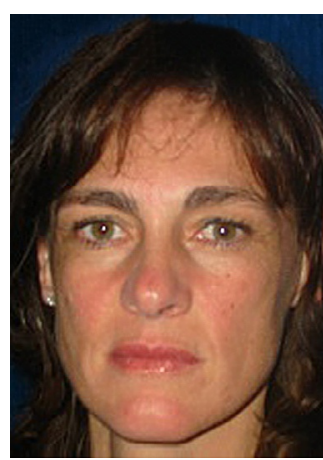

Agatha Bastida
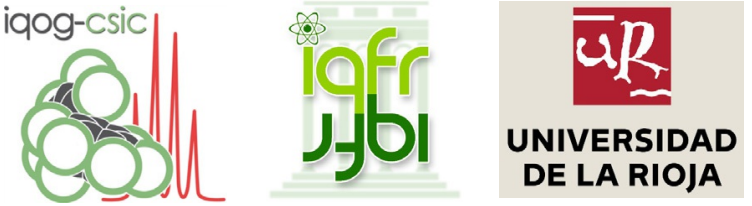

Invited for the cover of this issue are Andrés G. Santana, Carlos González, Juan Luis Asensio and co-workers at Instituto de Química Orgánica General, Instituto de Química-Física Rocasolano and Universidad de La Rioja. The image depicts drug selectivity using a metaphor of an arrow hitting a target. Read the full text of the article at 10.1002/chem.202005026.

What is the most significant result of this study?

While targeting DNA G-quadruplexes by small ligands holds significant promise in chemical biology, the development of strategies for the discrimination among alternative quadruplex fragments remains a significant challenge. Interestingly, we found that G-tetrads adjacent to duplex extensions present unusual electrostatic properties that can be exploited in the design of selective binders. This observation has inspired the design of cationic aromatic frameworks for the selective recognition of quadruplex-duplex junctions, thus providing a stepping stone to quadruplex discrimination.

How did the collaboration on this project start? How did each team member/collaborator contribute to the work? The reported work results from a long-term collaboration between three research groups with complementary expertise within the field of bioorganic chemistry. Namely, the group led by Asensio has focused its research activity in the analysis of molecular recognition processes of biological relevance and the design of strong/ selective binders, both for nucleic acids and in glycoscience. The Gonzalez group has been responsible for the elucidation of the first high-resolution three-dimensional structure of a quadruplexduplex junction complexed with a selective ligand, herein reported. Finally, the Corzana lab has lent computational support for the

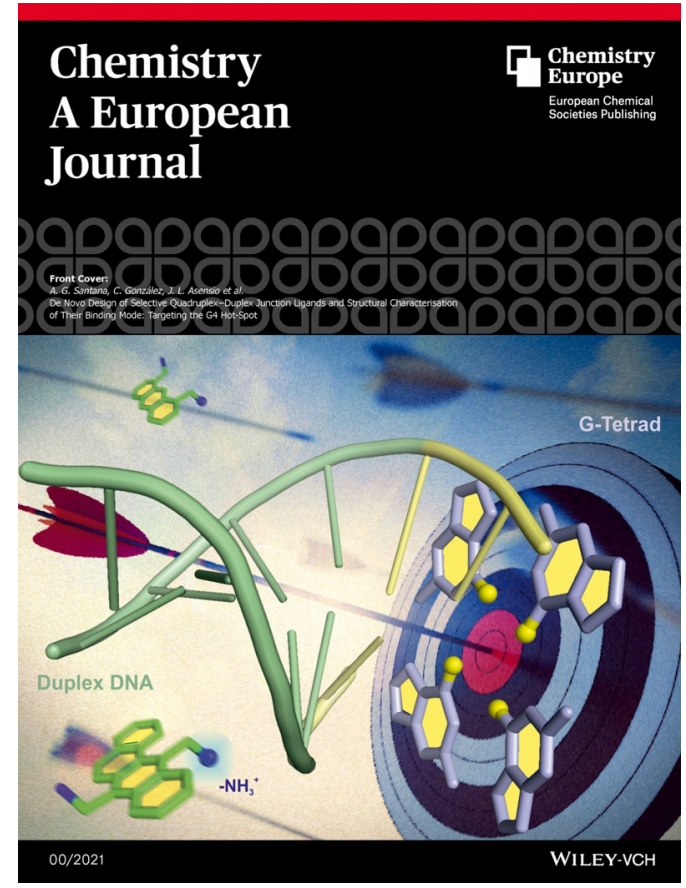


molecular dynamics simulations of the target DNA motifs assisting in the design of the proposed pharmacophore.

\section{What was the inspiration for this cover design?}

The cover image revolves around the concept of drug selectivity, exemplified by the straightforward metaphor of an arrow hitting the target. In this particular case, the structural features of this DNA hot-spot have been represented by a schematic duplex ex- tension aligned with an interfacial tetrad, as observed in targetable quadruplex-duplex junction architectures. In addition, examples of some of the designed ligands have been overlaid on top of the arrows to highlight the chemical features complying with the proposed pharmacophore. 